\title{
Evidence for azimuthal variations of the oxygen-abundance gradient tracing the spiral structure of the galaxy HCG $91 c^{\star}$
}

\author{
F. P. A. Vogt ${ }^{1, \star \star}$, E. Pérez ${ }^{2}$, M. A. Dopita ${ }^{3}$, L. Verdes-Montenegro ${ }^{2}$, and S. Borthakur ${ }^{4}$ \\ ${ }^{1}$ European Southern Observatory, Av. Alonso de Córdova 3107, 7630355 Vitacura, Santiago, Chile \\ e-mail: frederic.vogt@alumni .anu.edu. au \\ 2 Instituto de Astrofísica de Andalucía, CSIC, Apdo. Correos 3004, 18080 Granada, Spain \\ 3 Research School of Astronomy and Astrophysics, Australian National University, Canberra, Australia \\ ${ }^{4}$ Department of Physics and Astronomy, Johns Hopkins University, 3400 N. Charles Street, Baltimore, MD 21218, USA
}

Received 3 October 2016 / Accepted 23 December 2016

\begin{abstract}
Context. The distribution of elements in galaxies forms an important diagnostic tool to characterize these systems' formation and evolution. This tool is, however, complex to use in practice, as galaxies are subject to a range of simultaneous physical processes active from pc to kpc scales. This renders observations of the full optical extent of galaxies down to sub-kpc scales essential.

Aims. Using the WiFeS integral field spectrograph, we previously detected abrupt and localized variations in the gas-phase oxygen abundance of the spiral galaxy HCG 91c. Here, we follow-up on these observations to map HCG 91c's disk out to $\sim 2 R_{\mathrm{e}}$ at a resolution of $600 \mathrm{pc}$, and characterize the non-radial variations of the gas-phase oxygen abundance in the system.

Methods. We obtained deep MUSE observations of the target under $\sim 0.6$ arcsec seeing conditions. We perform both a spaxel-based and aperture-based analysis of the data to map the spatial variations of $12+\log (\mathrm{O} / \mathrm{H})$ across the disk of the galaxy.

Results. We confirm the presence of rapid variations of the oxygen abundance across the entire extent of the galaxy previously detected with WiFeS, for all azimuths and radii. The variations can be separated in two categories: a) localized and associated with individual H II regions; and b) extended over kpc scales, and occurring at the boundaries of the spiral structures in the galaxy.

Conclusions. Our MUSE observations suggest that the enrichment of the interstellar medium in HGC 91c has proceeded preferentially along spiral structures, and less efficiently across them. Our dataset highlights the importance of distinguishing individual star-forming regions down to scales of a few $100 \mathrm{pc}$ when using integral field spectrographs to spatially resolve the distribution of oxygen abundances in a given system, and accurately characterize azimuthal variations and intrinsic scatter.
\end{abstract}

Key words. ISM: abundances - HII regions - galaxies: groups: individual: HCG 91 - galaxies: ISM - galaxies: spiral

\section{Introduction}

The distribution within galaxies of the atomic elements created via stellar evolution is a key observable affected by the various processes associated with galaxy evolution. In principle, the physical mechanisms associated with specific processes affecting galaxies can be deciphered from a spatially-resolved characterization of the distribution of atomic elements heavier than Helium (located either in the gas phase or locked in subsequent generations of stars), provided that the spatial and temporal enrichment of the interstellar medium (ISM) in the system can be constrained. In practice, the co-existence of multiple evolutionary mechanisms effective on different spatial and temporal scales significantly complicates such an analysis for any given system. Evolutionary mechanisms include galactic gaseous outflows, gaseous inflows, stellar and gaseous radial migration (e.g., through the presence of a bar), gravitational interactions, mergers, accretion events, and other forms of perturbations, including strangulation, harassment, and stripping.

In recent years, integral field spectrographs (IFSs) have largely supplanted long-slit spectrographs in studies designed to characterize the abundance distribution of heavy elements

\footnotetext{
$\star$ The movie associated to Fig. 8 is available at http: //www . aanda.org

$\star \star$ ESO Fellow.
}

in galaxies. Among other benefits, the ability of IFS to measure abundances throughout the full two-dimensional extent of a galaxy (or a large part thereof) and detect azimuthal and radial trends has often been praised. In practice, the relatively small fields-of-view (FoVs) and/or large size (on-sky) of the spatial pixels (a.k.a spaxels) of many IFSs have so far restricted the feasibility of performing such combined analyses of both sub-kpc trends with larger kpc-scales variations in systems located beyond a few Mpc. Instead, gaseous abundance distributions are often characterized via the slope of the azimuth-averaged gradient; an approach usually driven by the lack of spatial resolution and/or signal-to-noise $(\mathrm{S} / \mathrm{N})$, but that also allows for a more straight-forward comparison between galaxies at higher redshift, modulo the issues inherent to comparing oxygen abundances derived using different methodologies (e.g., Kewley \& Ellison 2008; López-Sánchez et al. 2012).

The advent of the Multi-Unit Spectroscopic Explorer (MUSE) IFS has opened a new observational window as the combination of a relatively large FoV $(1 \times 1$ square arcmin) and small spaxels $(0.2 \times 0.2$ square arcsec $)$ coupled to the excellent seeing of Cerro Paranal is now allowing us to map galaxies out to 2-3 effective radius $\left(R_{\mathrm{e}}\right)$ and (simultaneously) down to sub-kpc scales out to distances of $\sim 100 \mathrm{Mpc}$. MUSE is effectively pushing outwards our ability to study galaxies as complex systems down to the crucial sub-kpc scales (the scale of 
individual $\mathrm{H}$ II region complexes); a feat previously restricted to galaxies closer-in via IFS with larger FoV such as PMAS/PPAK (e.g., via the PINGS and CALIFA surveys; Rosales-Ortega et al. 2010; Sánchez et al. 2012b,a, 2014) and VIRUS-P (e.g., via the VENGA survey; Blanc et al. 2013; Kaplan et al. 2016). This push is particularly interesting from a galaxy evolution perspective, as MUSE effectively allows the study of galaxies inside a wider range of environments, including that of compact groups and clusters, with unprecedented spatial resolution.

In this article, we present follow-up MUSE observations of the nearly face-on spiral galaxy HCG 91c. Initial observations of this galaxy with the Wide Field Spectrograph (WiFeS; Dopita et al. 2007, 2010) mounted on the ANU 2.3 m telescope (Mathewson et al. 2013) at Siding Spring observatory revealed spatially rapid and localized variations of the oxygen abundance in the system associated with at least one star-forming complex. HCG 91c is a member of a compact group of galaxies, which could indicate a possible influence of the environment on the chemical content within the galaxy. However, the WiFeS data were insufficient to distinguish between the possibility that gas of different metallicity had fallen in from another galaxy (or from the Intergalactic Medium), or whether the variation was caused by secular processes. The new MUSE data presented in Sect. 2 (thanks to the large FoV and $3 \times$ better spatial resolution of this instrument) now allow us to do this. Our analysis procedure is described in Sect. 3. We present our results in Sect. 4 and discuss them in Sect. 5. Our conclusions are summarized in Sect. 6.

\section{Observations and data reduction}

HCG 91c was observed during the second Science Verification run for MUSE (mounted at the Nasmyth B focus of the Unit 4 Yepun of the Very Large Telescope on Cerra Paranal), under program ID 60.A-9317(A) (P.I.: Vogt). The observation strategy for this program is described in detail in Vogt (2015), and summarized here for completeness. A total of 12 individual exposures (eleven of $1050 \mathrm{~s}$ and one of $525 \mathrm{~s}$ ) on-target separated in six observation blocks (OBs) were acquired over four distinct nights between August 20, 2014, and August 25, 2014. In each OB, two exposures on-target were surrounding the observation of a dedicated empty sky field located nearby. Each on-target exposure was reduced individually using the REFLEX (Freudling et al. 2013) MUSE workflow (v1.6), including the construction of the individual datacubes. Out of the 12 on-target exposures, 3 were acquired under seeing conditions $>0.8$ arcsec, measured from the reconstructed individual datacubes using a star in the FoV (and consistent with the reported values of the Slow Guiding System). The other 9 exposures were acquired under seeing conditions $<0.7$ arcsec: these 9 exposures were combined together to form the final datacube presented in this article via the dedicated REFLEX MUSE_EXP_COMBINE.XML workflow. The combined datacube thus corresponds to $8 \cdot 1050+1 \cdot 525=8925 \mathrm{~s}$ on-source, and the spatial full-width at half-maximum (FWHM) of stars in the FoV are measured to be 0.6 arcsec in the $V$-band. Spectrally, the cube extends from $4750 \AA$ to $9350 \AA$ in steps of $1.25 \AA$, with $319 \times 316$ spaxels, and the MUSE spectral resolution increasing from $R \cong 1700$ to $R \cong 3500$ between $4750 \AA$ and $9350 \AA$. The individual exposures were acquired at four distinct position angles $\left(\mathrm{PA} ; 0^{\circ}, 90^{\circ}, 180^{\circ}, 270^{\circ}\right)$ and with sub-arcsec spatial offsets to best remove the background-level artefacts associated with the 24 individual integral field units inside MUSE. The reduced datacube was uploaded to the ESO science archive

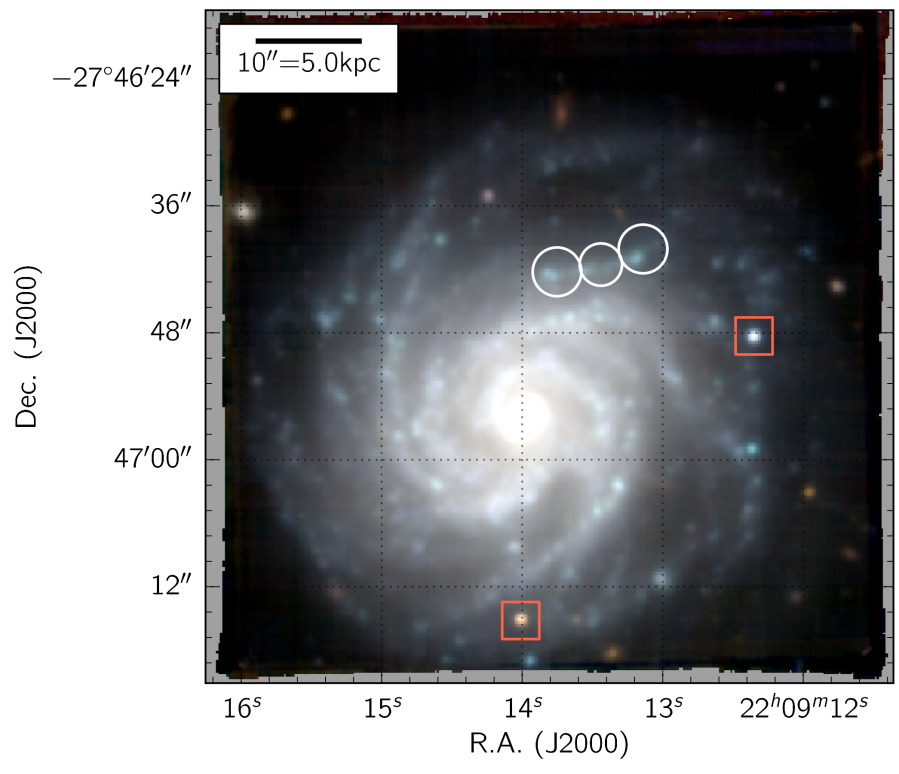

Fig. 1. Pseudo-RGB image of HCG 91c, where the $R, G$, and $B$ colors correspond to the sum of the reduced MUSE cube slices in the spectral ranges [7500 ̊; $9300 \AA$ ], [6000 ̊; $7500 \AA$ ], and [4800 respectively. The spatial FWHM of the datacube is $\sim 0.6$ arcsec in the $V$-band. The star-forming complexes found by Vogt et al. (2015) (then dubbed "C1", "C2", and "C3") to have discrepant oxygen abundances with respect to their immediate surroundings are marked with white circles. Two foreground stars (indicative of the spatial resolution of the data) are marked with red boxes.

facility following the recommendations of the Phase 3 stage of observations with ESO facilities ${ }^{1}$.

A pseudo-RGB image of the final datacube is presented in Fig. 1, where the $R, G$, and $B$-bands correspond to the sum of the cube slices in the spectral ranges [7500 $\mathrm{A} ; 9300 \AA$ ], [6000 $\AA$; $7500 \AA$ ], and [4800 $;$; $6000 \AA$ ], respectively. The spiral structure of the galaxy is readily evident, extending throughout the entire optical disk. Two foreground stars are visible at $\left[22^{\mathrm{h}} 09^{\mathrm{m}} 14.02\right.$; $\left.-27^{\circ} 47^{\prime} 15^{\prime \prime} .1\right]$ and $\left[22^{\mathrm{h}} 9^{\mathrm{m}} 12^{\mathrm{s}} .37 ;-27^{\circ} 46^{\prime} 48^{\prime \prime} .4\right]$, and provide a visual scale for the spatial resolution of the image. Numerous background galaxies are also visible as redder sources across the FoV. At the distance of HCG 91c (104 Mpc, see Table 1 and Vogt et al. 2015), 10 arcsec corresponds to $5 \mathrm{kpc}$.

\section{Data post-processing}

With $>10^{5}$ spectra in the final MUSE datacube, any manual data processing step becomes extremely costly time-wise. Each second invested for the analysis of a single spectra would immediately require $\sim 28 \mathrm{~h}$ to manually perform the same task for all the spaxels in the MUSE datacube (one after another). To circumvent this time sink (also associated with the processing of a large number of IFS observations from other instruments), several tools have been developed to process IFS data products in an automated fashion: recent examples include PYCASSO (Cid Fernandes et al. 2013), LZIFU (Ho et al. 2016), and PIPE3D (Sánchez et al. 2016b,a). For our analysis, we have developed our own post-processing tool called BRUTUS: a set of PYTHON modules designed to automatically process datacubes from integral field spectrographs. BRUTUS is hosted on Github, and is

\footnotetext{
https://www . eso.org/sci/observing/phase3.html
} 
Table 1. Basic characteristics of HCG 91c.

\begin{tabular}{lll}
\hline \hline Property & Value & Reference \\
\hline Names & HCG 91c & \\
& ESO 467 - G 013 & \\
RA [J2000] & $22^{\mathrm{h}} 09^{\mathrm{m}} 07.7^{\mathrm{s}}$ & \\
Dec [J2000] & $-27^{\circ} 48^{\prime} 34^{\prime \prime}$ & \\
Redshift & 0.024377 & Vogt et al. (2015) \\
Distance & $104 \mathrm{Mpc}$ & \\
Spatial scale & $504 \mathrm{pc}$ arcsec & \\
$R_{25}$ & $26.75 \pm 3.25 \mathrm{arcsec}^{-1}$ & de Vaucouleurs et al. (1991) \\
Rotation velocity & $100 \pm 11 \mathrm{~km} \mathrm{~s}^{-1}$ & Vogt et al. (2015) \\
$\quad$ (at radii $>22 \mathrm{kpc})$ & & \\
Star-formation rate & $2.19 M_{\odot} \mathrm{yr}^{-1}$ & Bitsakis et al. (2014) \\
& $2.10 \pm 0.06 M_{\odot} \mathrm{yr}^{-1}$ & Vogt et al. (2015) \\
Stellar mass & $1.86 \times 10^{10} M_{\odot}$ & Bitsakis et al. (2014) \\
H I mass & $2.3 \times 10^{10} M_{\odot}$ & Borthakur et al. (2010) \\
\hline
\end{tabular}

made freely available to the community ${ }^{2}$. It is designed with a modular structure in mind (inspired by PYWIFES; Childress et al. 2014b,a), allowing users to choose which processing steps are to be run (or not). A detailed description of the code is outside the scope of this article, but, for completeness, we list the specific steps employed to process the datacube of HCG 91c in Appendix A.

The emission line flux maps for $\mathrm{H} \alpha$ and $[\mathrm{O} \mathrm{III}] \lambda 5007$ constructed using BRUTUS are presented in Fig. 2. In this work, we restrict our analysis to spaxels with $S / N(\mathrm{H} \alpha, \mathrm{H} \beta) \geq 5$ and $S / N \geq 3$ for all other lines: a good detection of the first two hydrogen Balmer line ensures reliable measurements of the tied velocity and velocity dispersions, hence leading to stable fits for the other lines for $S / N s \geq 3$. A significant detection of the first two hydrogen Balmer line is also essential to ensure a reliable correction of the extragalactic attenuation on a spaxel-by-spaxel basis. In the present case, the extragalactic attenuation is corrected with BRUTUS via the $\mathrm{H} \alpha$ to $\mathrm{H} \beta$ line flux ratio and the theoretical model of a turbulent dust screen from Fischera \& Dopita (2005), with $R_{V}=3.08$ and $R_{V}^{A}=4.3$ which, in practice, results in a correction function very similar to that of Calzetti et al. (2000) across the MUSE spectral range.

Whereas a spaxel-based analysis best exploits the high spatial resolution of MUSE observations, the $\mathrm{S} / \mathrm{N}$ in the strong emission lines (and in particular $\mathrm{H} \beta$ ) is too little for numerous star-forming regions in the outskirts of the galaxy to perform a reliable analysis. Hence, we supplement the spaxelbased approach with an aperture-based one. Namely, we use BRUTUS to detect HII regions in the datacube automatically, by detecting local maxima in the integrated $\mathrm{H} \alpha$ flux map (see Fig. 2). Our approach is somewhat reminiscent of that adopted by Sánchez et al. (2012b), but our codes are entirely different in practice (see Appendix A for details). For HCG 91c, we defined 556 circular apertures associated with individual local maxima in the $\mathrm{H} \alpha$ flux map, all of which are presented in Fig. 3.

From the set of strong emission line fluxes derived from both the spaxel-based and aperture-based spectral fitting, we derive estimates of the gas-phase oxygen abundance $12+\log (\mathrm{O} / \mathrm{H})$ and ionization parameter $\log (\mathrm{Q})$. Here, we rely on the PYQZ PYTHON module v0.8.1, first introduced (as v0.4) in Dopita et al. (2013) with the full propagation of observational errors subsequently implemented in v0.6 (see Appendix B in Vogt et al. 2015). The latest embodiment of the code relies on the photo-ionization

\footnotetext{
2 http://fpavogt.github.io/brutus
}

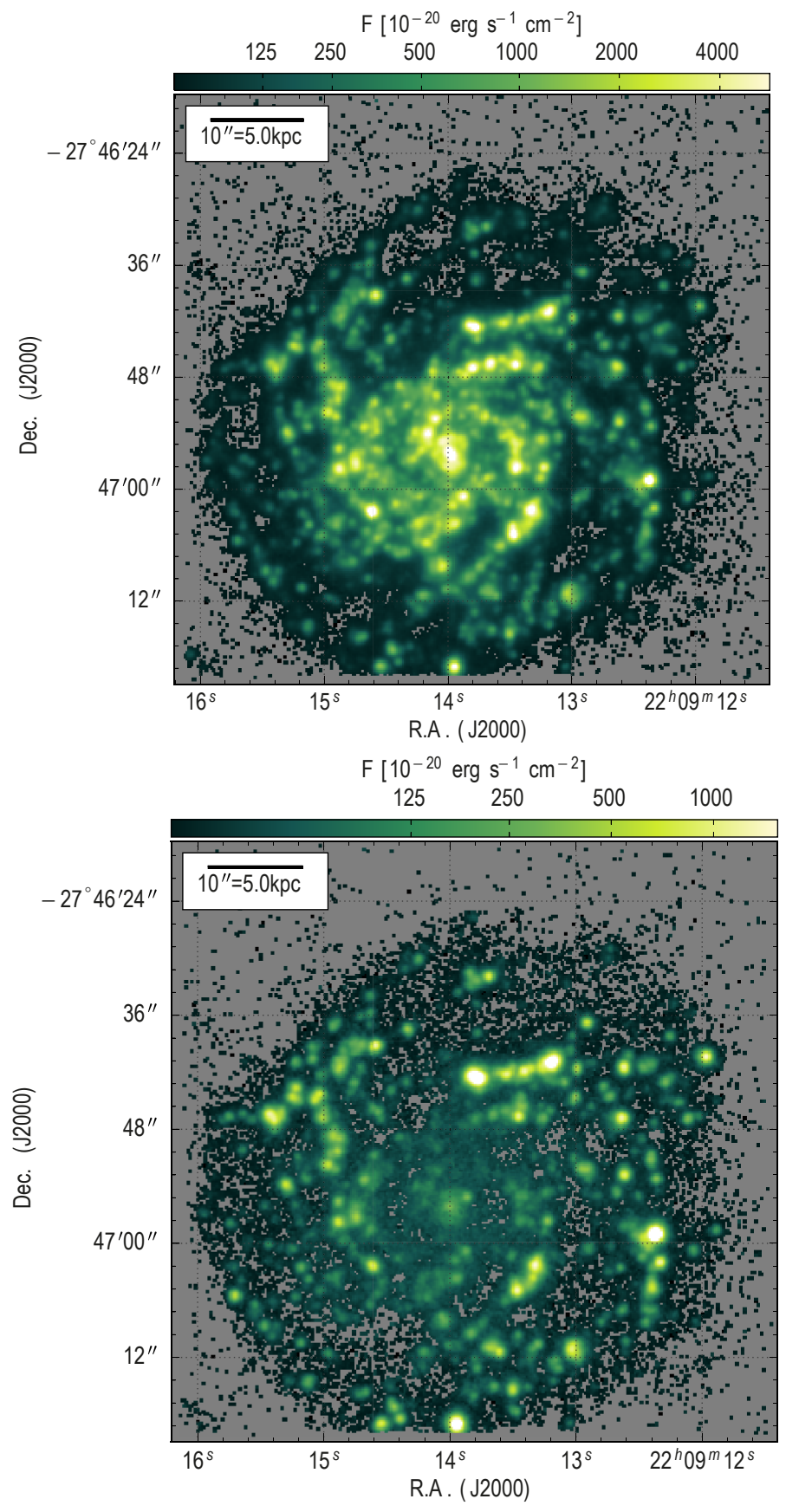

Fig. 2. $\mathrm{H} \alpha$ (top) and [O III] $\lambda 5007$ flux map of HCG 91c. The emission lines were fitted with tied Gaussian components in each spaxel using BRUTUS. For the $\mathrm{H} \alpha$ map, all spaxels with $S / N \geq 5$ are shown. For the [O III] $\lambda 5007$ map, all spaxels with $S / N \geq 3$ are shown.

models from the MAPPINGS $\mathrm{V} \operatorname{code}^{3}$ (Sutherland et al., in prep.), and is now hosted on a dedicated Github repository ${ }^{4}$. The principle and physical basis of the code however remains unchanged with respect to Dopita et al. (2013); specific line ratio spaces, in which the photo-ionization model grids are flat, without wraps and cleanly allow to disentangle the influence of $12+\log (\mathrm{O} / \mathrm{H})$ and $\log (\mathrm{Q})$, are used to derive estimates of these two parameters associated with a given set of strong line fluxes. For this analysis, we rely on the plane-parallel photo-ionization models with $P_{k}=5.0$ and $\kappa=\infty$ (i.e., a Maxwell-Boltzmann energy

\footnotetext{
3 https://miocene.anu.edu.au/Mappings/

4 http://fpavogt.github.io/pyqz
} 


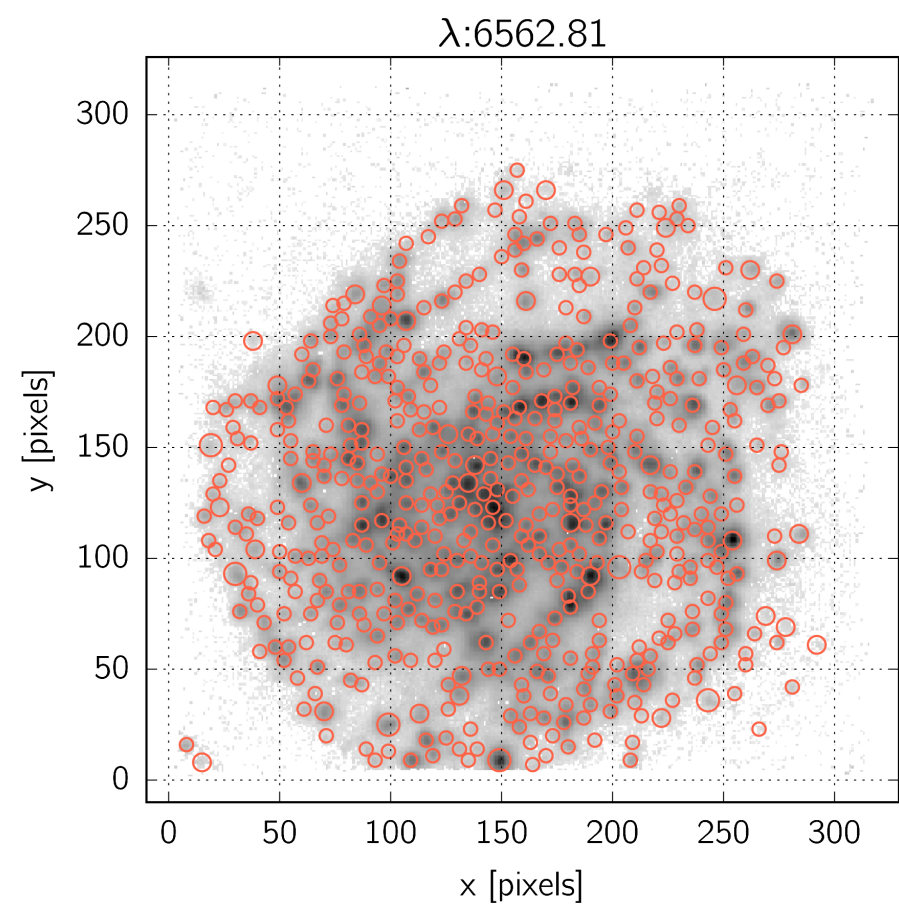

Fig. 3. H $\alpha$ flux map of HCG 91c overlaid with the 556 apertures associated with local maxima. The apertures were first identified automatically by BRUTUS, and subsequently inspected and adjusted manually using a dedicated interactive module inside the code.

distribution for the electrons; see Nicholls et al. 2012, 2013). The propagation of observational errors is achieved through the generation of 400 (in this case) random realizations of line fluxes according to the error distribution of the measured lines (assumed to be Gaussian), and the subsequent reconstruction of the full probability density function in the $12+\log (\mathrm{O} / \mathrm{H})$ versus $\log (\mathrm{Q})$ plane from these 400 individual estimates, using Kernel Density Estimate (KDE) techniques. For this work, we adopt the MAPPINGS 5.1 models with the local Galactic concordance (LGC) abundance set, in which the local region reference abundance corresponds to $12+\log (\mathrm{O} / \mathrm{H})=8.76$ (Nicholls et al. 2016).

Several of the PYQZ diagnostic grids involve the [O II] $\lambda \lambda 3726,3729$ emission lines, which at the redshift of HCG 91c do not fall within the MUSE spectral range. Here, we derive our estimates of $12+\log (\mathrm{O} / \mathrm{H})$ and $\log (\mathrm{Q})$ from the combination of the following two diagnostic grids that do not employ that line:

$$
\begin{array}{lll}
\log \left(\frac{[\mathrm{N} \mathrm{II}] \lambda 6583}{[\mathrm{~S} \mathrm{II}] \lambda \lambda 6716,6731}\right) & \text { vs. } & \log \left(\frac{[\mathrm{O} \text { III }] \lambda 5007}{\mathrm{H} \beta}\right), \text { and } \\
\log \left(\frac{[\mathrm{N} \mathrm{II}] \lambda 6583}{[\mathrm{~S} \mathrm{II}] \lambda \lambda 6716,6731}\right) & \text { vs. } & \log \left(\frac{[\mathrm{O} \text { III }] \lambda 5007}{[\mathrm{~S} \mathrm{II}] \lambda \lambda 6716,6731}\right) .
\end{array}
$$

The distribution of each of the 556 apertures in both line ratio planes is shown in Fig. 4. Both diagnostics are in excellent agreement throughout most of the abundance range, except for the star-forming regions with $12+\log (\mathrm{O} / \mathrm{H}) \lesssim 8.1$, which tend to be less enriched according to the first diagnostic grid. This mismatch is most likely impacted by problems within the theoretical models at the low-abundance end, rather than fully by a miscorrection of the extragalactic reddening (which is nonetheless likely to be playing a role as well). Indeed, a reddening correction issue would have likely affected all apertures, which is not seen here.

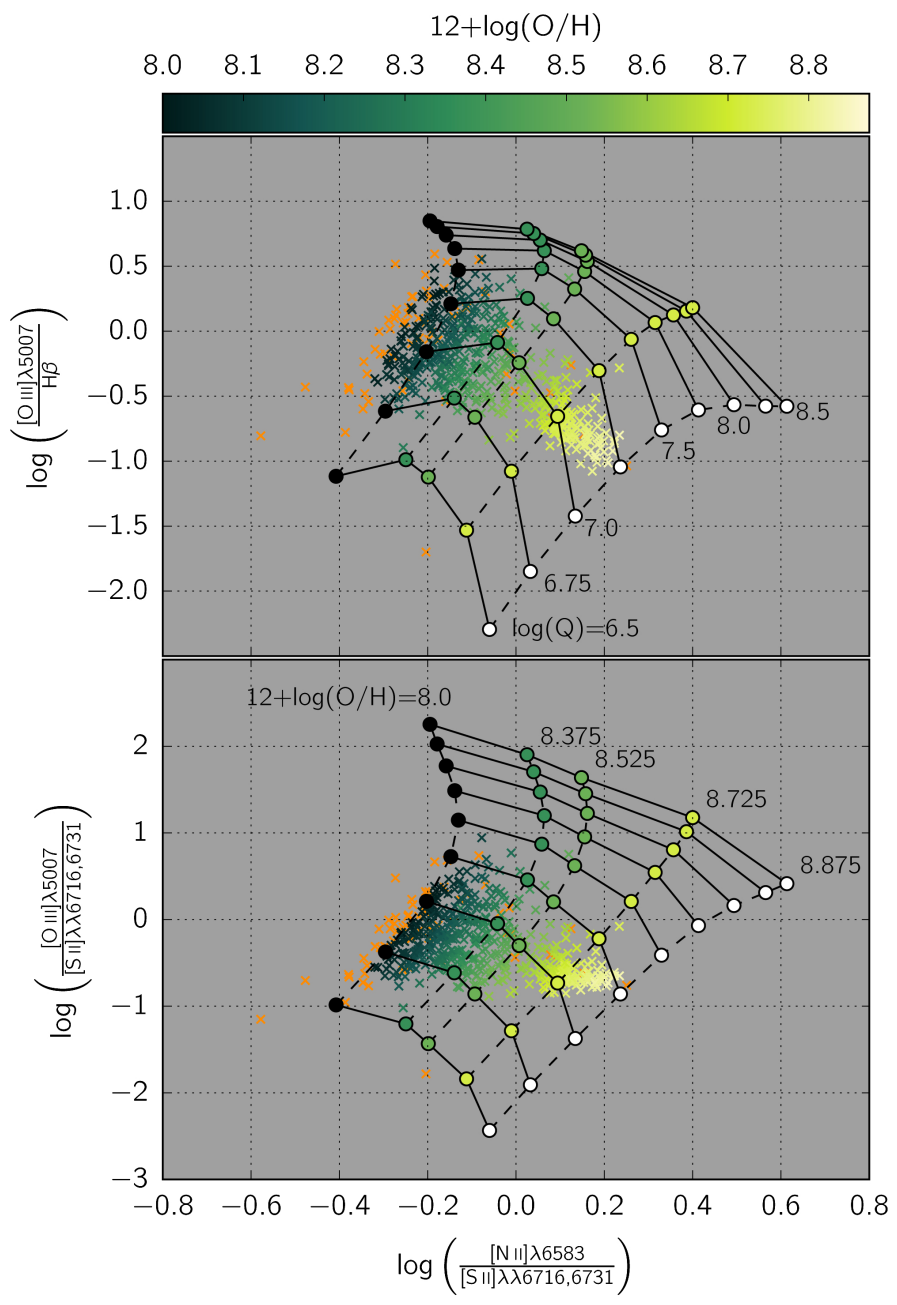

Fig. 4. Emission line ratio diagnostic grids from the MAPPINGS 5.1 photo-ionization code for a plane-parallel geometry with $P_{k}=5.0$ and $\kappa=\infty$. Each circle corresponds to one simulation with a specific abundance and ionization parameter. Small crosses indicate the location of each of the 556 circular apertures, color-coded as a function of the combined (where suitable) abundance value derived from both diagnostics. Orange crosses mark the apertures for which PYQZ could not derive a reliable abundance: either from an overly large discrepancy between both grids, or because the estimates fall outside both grids.

A detailed investigation of this mismatch - complicated by the lack of the [O II] $\lambda \lambda 3726,3729$ in the MUSE spectral range is beyond the scope of this article. It may, for example, be that our model, with $P_{k}=5.0$, may not be suitable for these starforming regions, and/or this mismatch may simply reflect genuine limitations of the MAPPING $\mathrm{V}$ models at the low-abundance end. In this analysis, we choose to also use the apertures for which only one diagnostic grid returns a suitable estimate, noting and stressing that this choice does not affect our conclusions. In particular, we note that the trends discussed in the next Section are present when considering either diagnostic grids separately, or together.

\section{Results}

The maps of $12+\log (\mathrm{O} / \mathrm{H})$ and $\log (\mathrm{Q})$ for both the spaxel-based case and the aperture-based case are presented in Figs. 5 and 6. The spatial resolution of these maps (and the gain provided by MUSE) can be compared with those obtained with the WiFeS 
F. P. A. Vogt et al.: The azimuthal variations of the oxygen-abundance gradient in HCG 91c
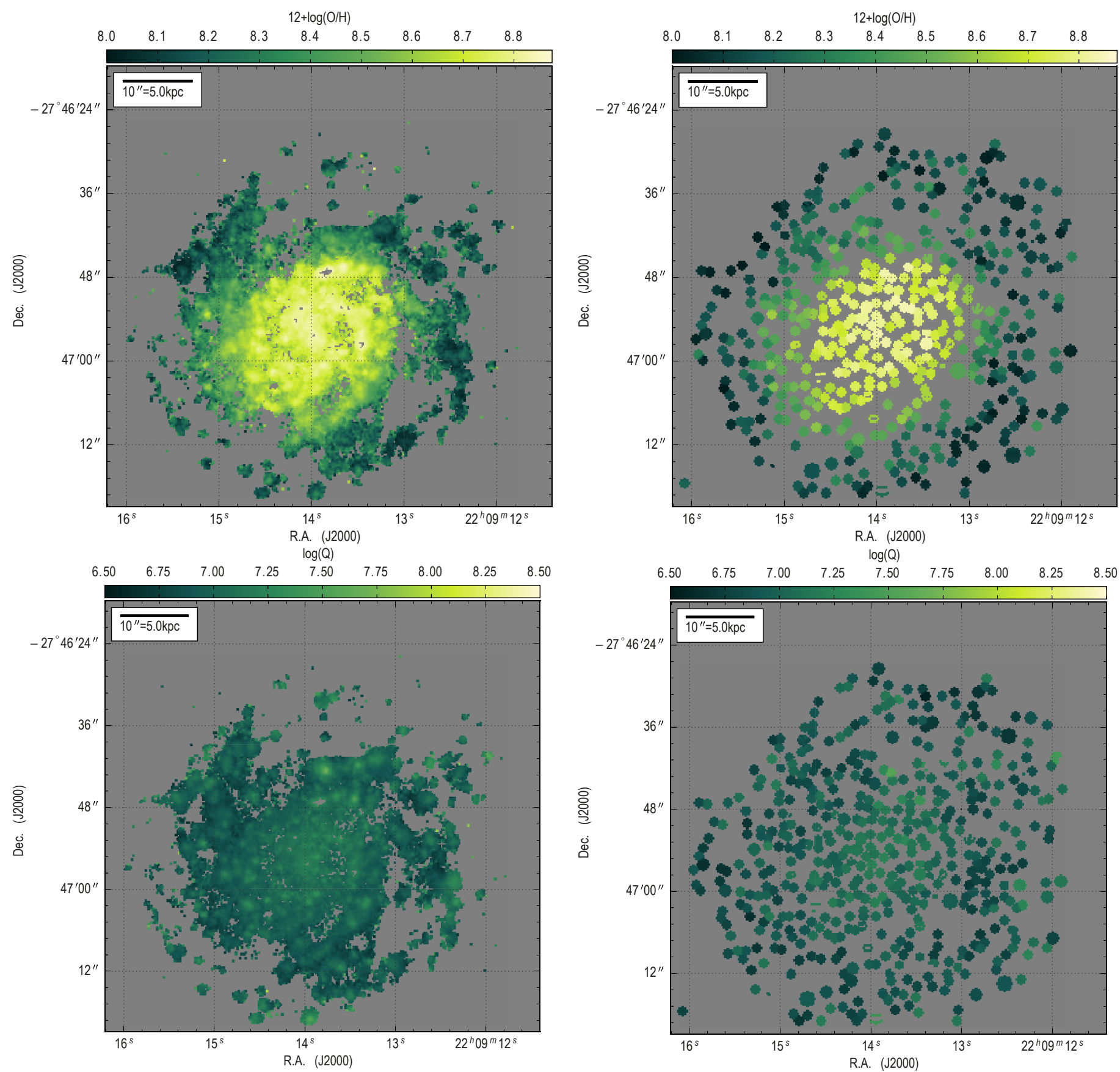

Fig. 5. Spaxel-based map of the oxygen abundance (top) and ionization parameter (bottom) in HCG 91c, constructed using the BRUTUS and PYQZ codes. The color bars span the full range of values covered by the MAPPINGS V simulations, highlighting the large range of oxygen abundances and narrower range of ionization parameters throughout the galaxy.

integral field spectrograph described in Vogt et al. (2015); the general features identified with WiFeS remain (in particular the sharp decrease in the oxygen abundance at $\sim 6 \mathrm{kpc}$ North from the galaxy center), but the significant improvement in the spatial resolution (both in terms of seeing and spaxel size) also allows us to better resolve structures within the disk, as well as to detect and characterize H II regions at larger radii than with WiFeS.

Local enhancements of the ionization parameter are present throughout the disc, and are associated with individual star-forming complexes. The largest values of $\log (\mathrm{Q})$ are found

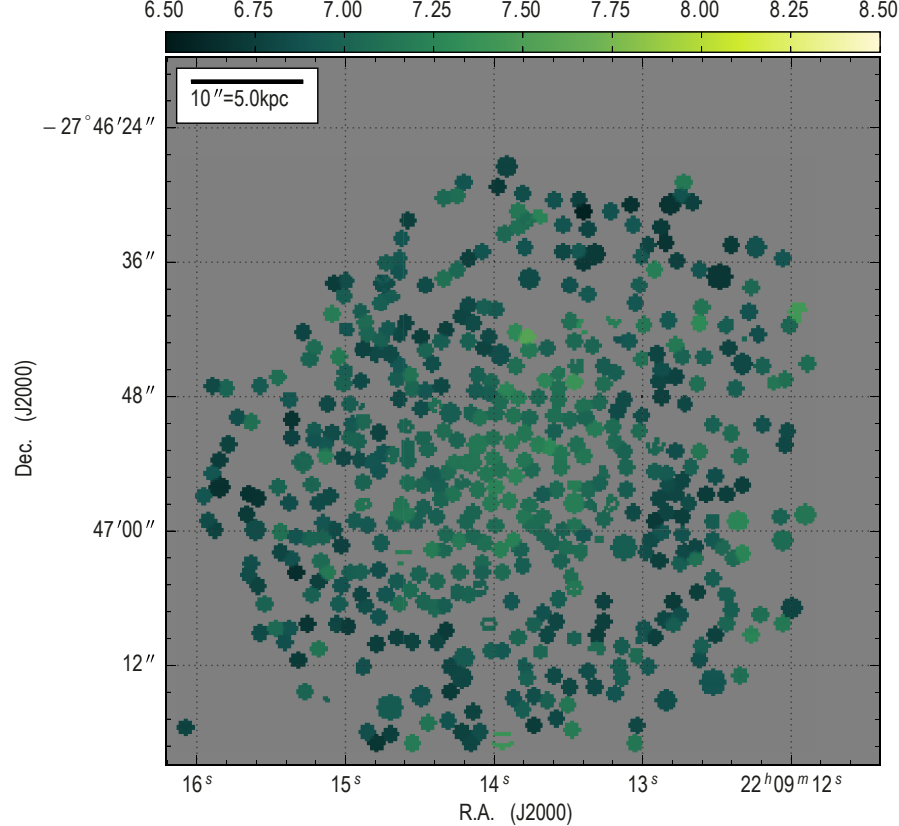

Fig. 6. Same as Fig. 5, but for the 556 apertures associated with local maxima in the $\mathrm{H} \alpha$ flux map of the galaxy, identified with BRUTUS.

in the outskirts of the galaxy, beyond the effective radius $\left(R_{\mathrm{e}}=\right.$ $5.1 \mathrm{kpc})$. In terms of the oxygen abundance, the picture revealed by MUSE is clearly more complex than that discussed by Vogt et al. (2015) from the WiFeS observations of the system.

In Figs. 7 and 8, we present the oxygen abundance gradient of HCG 91c observed by MUSE; both global and along specific azimuths. When considering all spaxels and/or apertures at once, the oxygen abundance in HCG 91c displays a linear decline of $-0.082 \pm 0.001 \mathrm{dex} \mathrm{kpc}^{-1} \equiv-0.418 \pm 0.005 \mathrm{dex} R_{\mathrm{e}}^{-1}$ from $1 R_{\mathrm{e}}(5.1 \mathrm{kpc})$ to $2 R_{\mathrm{e}}(10.2 \mathrm{kpc})$, with a flattening inwards of $\sim 0.8 R_{\mathrm{e}}(4 \mathrm{kpc})$, and (possibly) outwards of $2.1 R_{\mathrm{e}}(11 \mathrm{kpc})$; a trend already detected with WiFeS (Vogt et al. 2015). Modulo a reduced scatter, both the spaxel-based and aperture-based 

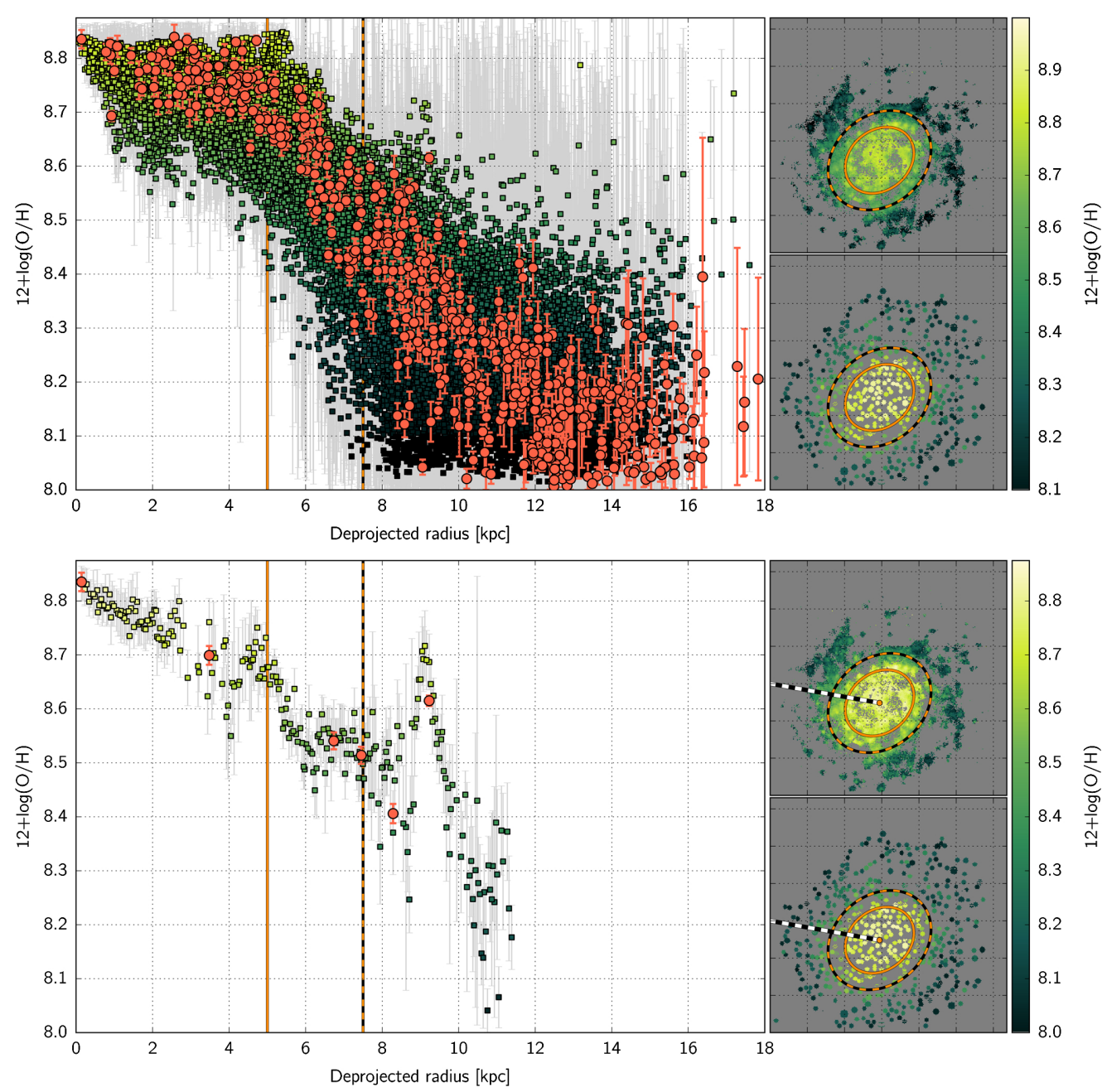

Fig. 7. Global oxygen abundance gradient (top panel) and for regions within 0.3 arcsec of the specific azimuth $79.5^{\circ}$ East-of-North (i.e., the area between the white \& black dashed line). Individual spaxels are shown as colored squares with the associated $1 \sigma$ errors in gray. Measurements for apertures (with associated $1 \sigma$ errors) are shown in red. Whereas, globally, the gradient in HCG 91c can be described as linear with a flattening inwards of $4 \mathrm{kpc}$ and (possibly) outwards of $11 \mathrm{kpc}$, the trends are highly non-linear for individual azimuths. Rapid and localized variations, detected both in individual spaxels and integrated apertures alike are present at all radii. Ellipses marking the deprojected effective radius $R_{\mathrm{e}}$ (5.1 kpc, orange) and $1.5 R_{\mathrm{e}}$ (dashed-orange) radius from the galaxy center are shown in all oxygen abundance maps (both spaxel-based and aperture based).

gradients display similar trends, indicative that (as one would expect) the distance to HCG 91c is large enough not to affect a spaxel-based approach through the resolution of the temperature structures of H II regions. The consistency between the spaxelbased and aperture-based approaches also indicates that the presence of diffuse ionized gas (DIG) in HCG 91c is not affecting our ability to derive reliable estimates of the $12+\log (\mathrm{O} / \mathrm{H})$ and $\log (\mathrm{Q})$. We note that large areas void of clearly-identified starforming regions (e.g., the inter-arm region to the South-West of the galaxy center) - and thus possibly dominated by DIG emission - have too little $\mathrm{S} / \mathrm{N}$ to be processed reliably by PYQZ.

A flattening of the oxygen abundance gradient beyond $2 R_{\mathrm{e}}$ has been identified as a generic feature in CALIFA galaxies (Sánchez et al. 2014; Sánchez-Menguiano et al. 2016b; Zinchenko et al. 2016), with the flattening of the gradient inside $\sim 0.5 R_{\mathrm{e}}$ identified primarily in galaxies with stellar masses $\log \left(M_{\star} / M_{\odot}\right) \geq 10.5$. The gradient slope derived by PYQZ for HCG $91 \mathrm{c}$ is somewhat steeper than the universal gradient measured with CALIFA galaxies $\left(-0.075 \operatorname{dex} R_{\mathrm{e}}^{-1}\right.$ with a scatter of $0.016 \mathrm{dex} R_{\mathrm{e}}^{-1}$, see Sánchez-Menguiano et al. 2016b). This mismatch is due to the different techniques used to derive the oxygen abundance values (as illustrated in Fig. 9): compared to the O3N2 calibrations of Marino et al. (2013), PYQZ and the underlying MAPPINGS $\mathrm{V}$ photo-ionization models return a wider range of abundances in HCG 91c, leading to a steeper gradient. Taking this scaling difference into account, the oxygen abundance gradient in HCG 91c (between $1 R_{\mathrm{e}}$ and $2 R_{\mathrm{e}}$ ) is consistent with the universal gradient reported by Sánchez-Menguiano et al. (2016b).

Our ability to spatially resolve sub-kpc scales for all azimuths in HCG 91c also reveals additional features of the oxygen abundance distribution throughout the galaxy, beyond the existence of a global gradient. Two distinct types of behaviors are present: first, on sub-kpc-scales, and second on kpc-scales. We describe them separately in the following sections.

\subsection{The sub-kpc-scale variations of $12+\log (\mathrm{O} / \mathrm{H})$}

The global gradient visible in Fig. 7 (top) contains a vertical scatter of $\sim 0.2$ dex in the oxygen abundance present at all radii in HCG 91c. When considering only the aperture-based measurements, the vertical scatter remains of the order of 

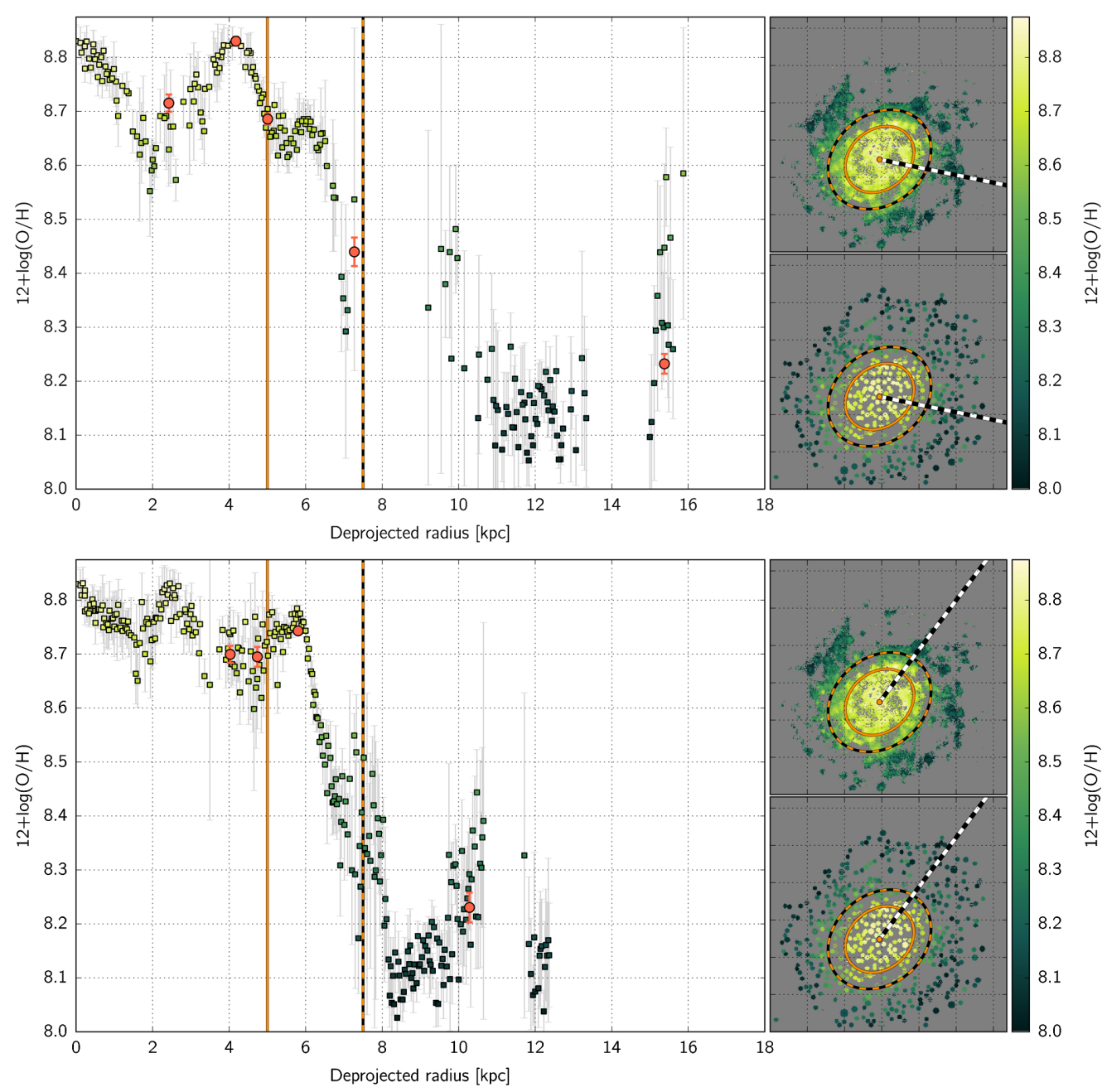

Fig. 8. Same as Fig. 7 but for azimuths $258.0^{\circ}$ (top) and $322.5^{\circ}$ (bottom) East-of-North, respectively. For completeness, the individual oxygen abundance gradients of HCG 91c extracted for all azimuths in steps of $0.5^{\circ}$ have been stacked into a movie available online.

$\sim 0.15$ dex despite intrinsically smaller measurement errors. It becomes evident that this vertical scatter is real (rather than related to measurement errors) when inspecting the oxygen abundance gradient as a function of the azimuth, as illustrated by the lower panel of Fig. 7 and those in Fig. 8. Restricting the azimuthal range of the gradient diagram reveals the presence of spatially localized and coherent variations in the measured oxygen abundances. The variations are of the order of $0.1-0.2$ dex over distances of $\leq 1 \mathrm{kpc}$, significant at more than $5 \sigma$ for most cases, and detected both in individual spaxels and integrated apertures (both consistent with one another). These rapid variations can be visually identified in the maps of the oxygen abundance (see Fig. 5); the clearest example of which is located 15 arcsec East of the galaxy center.

\subsection{The kpc-scale variations of $12+\log (\mathrm{O} / \mathrm{H})$}

An alternative approach to visualizing the gaseous oxygen abundance distribution in HCG 91c is presented in Fig. 10, where we de-wrapped the oxygen abundance gradient on the azimuth-distance plane. In this projection, all points at a given height are located at the same distance from the galaxy center. This projection facilitates the identification and inspection of spiral structures beyond the effective radius, at the cost of lesser clarity closer to the galaxy center (stretched horizontally across the diagram).

Large kpc-scale coherent trends in the oxygen abundance distribution pattern are present throughout HCG 91c, in addition to the spatially localized variations described previously. These features, the most prominent of which are traced by purple lines in Fig. 10, are located at the edges of the spiral arms of the galaxy, as illustrated in Fig. 11. In other words, the variations in the gaseous oxygen abundance are sharper and more abrupt when moving across the spiral structure, and more gradual when moving along the spiral arms. The rapid variation of the oxygen abundance measured with WiFeS to the North of the galaxy center (Vogt et al. 2015) corresponds to the (then) unresolved crossing of a spiral structure at that location, which the MUSE datacube reveals is not an isolated case, but rather the sharpest example of a behavior present at all azimuths.

\section{Discussion}

The detection of sub-kpc-scale, spatially localized variations of the oxygen abundance in HCG 91c, a spiral galaxy, is not excessively suprising. In near-by grand design spirals for example, Bresolin et al. (2009) report that the intrinsic scatter of the oxygen abundance is of the order of 0.2 dex throughout the disk. Croxall et al. (2016) measure intrinsic dispersion 


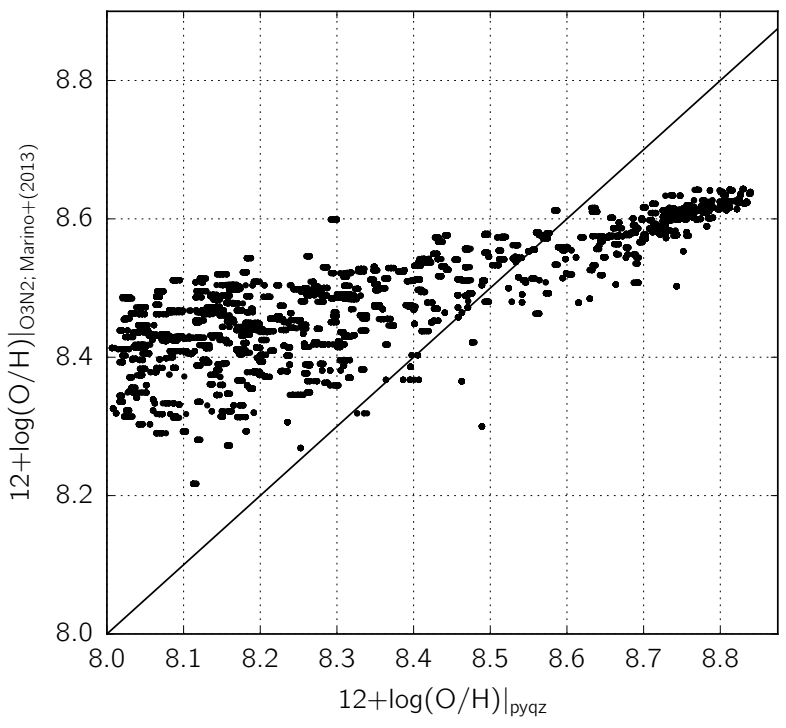

Fig. 9. Comparison of the oxygen abundance values derived via PYQZ v0.8.1 (i.e., using the MAPPINGS $\mathrm{V}$ photo-ionization models; this work) and the O3N2 calibration of Marino et al. (2013), as employed by Sánchez-Menguiano et al. (2016b) for all the aperture spectra in HCG 91c. PYQZ estimates span a wider range of oxygen abundances, meaning that PYQZ-derived gradient slopes are steeper than those derived by Sánchez-Menguiano et al. (2016b). Despite a scatter of $\sim 0.1$ dex at the lower-abundance end, the correspondence between the two techniques remains nonetheless linear over the span of oxygen abundances in HCG 91c.

in $12+\log (\mathrm{O} / \mathrm{H})$ of $0.074 \mathrm{dex}$ in NGC 5457 (M101, see also Kennicutt \& Garnett 1996). In the same galaxy, Li et al. (2013) report two cases of locally lower and higher oxygen abundances for two H II regions compared to their immediate surroundings. Rosolowsky \& Simon (2008) detect a scatter of 0.21 dex in M33 (but see also Bresolin 2011, that from a sample of $25 \mathrm{H} \mathrm{II} \mathrm{re-}$ gions only finds a scatter of $0.06 \mathrm{dex}$ ). Sanders et al. (2012) find localized variations of the oxygen abundance associated with individual star-forming regions of the order of 0.3 dex in M31, and Berg et al. (2015) report a scatter of 0.165 dex in NGC 0628 (see also Rosales-Ortega et al. 2011). Although caution must certainly be used when comparing these different studies with one another, the presence of localized variations of the oxygenabundance of star-forming regions with respect to their immediate surroundings within spiral galaxies remains unequivocal. From this perspective, the case of HCG 91c clearly illustrates the importance of characterizing the gaseous oxygen abundance throughout the entire optical discs of galaxies while at the same time distinguishing individual star-forming complexes within, in order to capture both pc-scale and kpc-scale trends. For long slit studies of nearby systems, the case of HCG 91c reinforces the importance of selection bias in deriving local abundance scatter and global trends from a handful of locations within a galaxy's disc. Ideally, such studies should characterize different galacticcentric radii (including both in and between spiral arms) and azimuths using not one but several $\mathrm{H}$ II regions in the immediate vicinity of one another for any given location.

The full mapping of the oxygen throughout HCG 91c also reveals large scale azimuthal variations unambiguously associated with galactic structures (i.e., the spiral arms). Observationally, similar behaviors have already been reported for different systems, with varying degrees of certainty. For example, Sánchez et al. (2015) detected (with MUSE) possible azimuthal variations in the oxygen abundance gradient of NGC 6754 associated with the spiral pattern of this galaxy (see also the recent re-analysis of this target by Sánchez-Menguiano et al. 2016a). In NCG 5457, Croxall et al. (2016) identify a mild correlation of the oxygen abundance distribution with the spiral arms from a sample of approximately $100 \mathrm{H} \mathrm{II} \mathrm{regions} \mathrm{observed} \mathrm{with}$ the Large Binocular Telescope. Such a correlation suggests a picture where ISM enrichment occurs preferentially along the spiral structures rather than across them. HCG 91c lies within a compact group, but its very regular HI envelope is a clear indication that the galaxy has not yet interacted strongly with the group environment (Vogt et al. 2015). It thus appears more likely that the larger-scale abundance variations tracing the spiral pattern in HCG 91c are an intrinsic behavior of the system, rather than a consequence of the environment. In particular, a (set of) selfdriven mechanism(s) appears more plausible than the different environment-driven hypothesis discussed in Vogt et al. (2015).

Still, the question remains as to what extent the gas phase abundance variations in HCG 91c (local or global) are affected by dynamics, and to what extent they reflect an enhanced star formation efficiency within the spiral structure (that keeps the metals in place). Theoretically, Grand et al. (2016) presents evidence for the ability of spiral patterns to transfer comparatively more metal-rich stars from the inner regions of the galaxy to the outer parts along the spiral arms (see also Grand et al. 2012). As these enriched stars end their lives in comparatively less enriched regions of the galaxy, they could contribute to a local enhancement of the ISM at larger radii. Alternatively, spiral arms in HCG 91c might have merely been acting as gravitational sinks, effectively trapping heavy elements while favoring star-formation activity, thus leading to the present distribution of the oxygen abundance in HCG 91c. Differentiating between these scenarios (and others) would require a better understanding of the nature of the spiral structure in HCG 91c (including its stability and coherence over time, see e.g., Dobbs \& Baba 2014; Baba et al. 2015). From that perspective, a combined gas +stars analysis of the MUSE observations of HCG 91c bridging gaseous and stellar abundance (and kinematics) appears very indicated, but beyond the scope of this article.

\section{Conclusions}

Here, we present MUSE observations of HCG 91c, as a followup of observations acquired with the WiFeS integral field spectrograph (Vogt et al. 2015). Using the new BRUTUS tool, designed to process the data cubes of integral field spectrographs, we have have measured the oxygen abundance and ionization parameter associated with star-forming regions throughout the disc of HCG 91c out to $\sim 2 R e$, using both a spaxel-based and aperture-based approach. We confirm the presence of rapid abundance variations in the galaxy initially detected with WiFeS. These variations can be separated into two distinct types. First, sub-kpc-scale variations associated with individual star-forming regions, and second, kpc-scale variations correlated with the spiral structure of the galaxy (specifically with the boundaries of spiral arms). The kpc-scale variations thus provide observational evidence that ISM enrichment is preferentially occurring along the spiral structure of HCG 91c, and less easily across it. As per the sub-kpc-scale variations of the oxygen abundance in HCG 91c, they are reminiscent of the behavior of star-forming regions observed in nearby grand design spirals.

The MUSE observations of HCG 91c confirm the unique ability of this instrument to spatially resolve oxygen abundance gradients, characterize intrinsic scatter and map non-radial variations of the abundance of HII regions in galaxies. The 


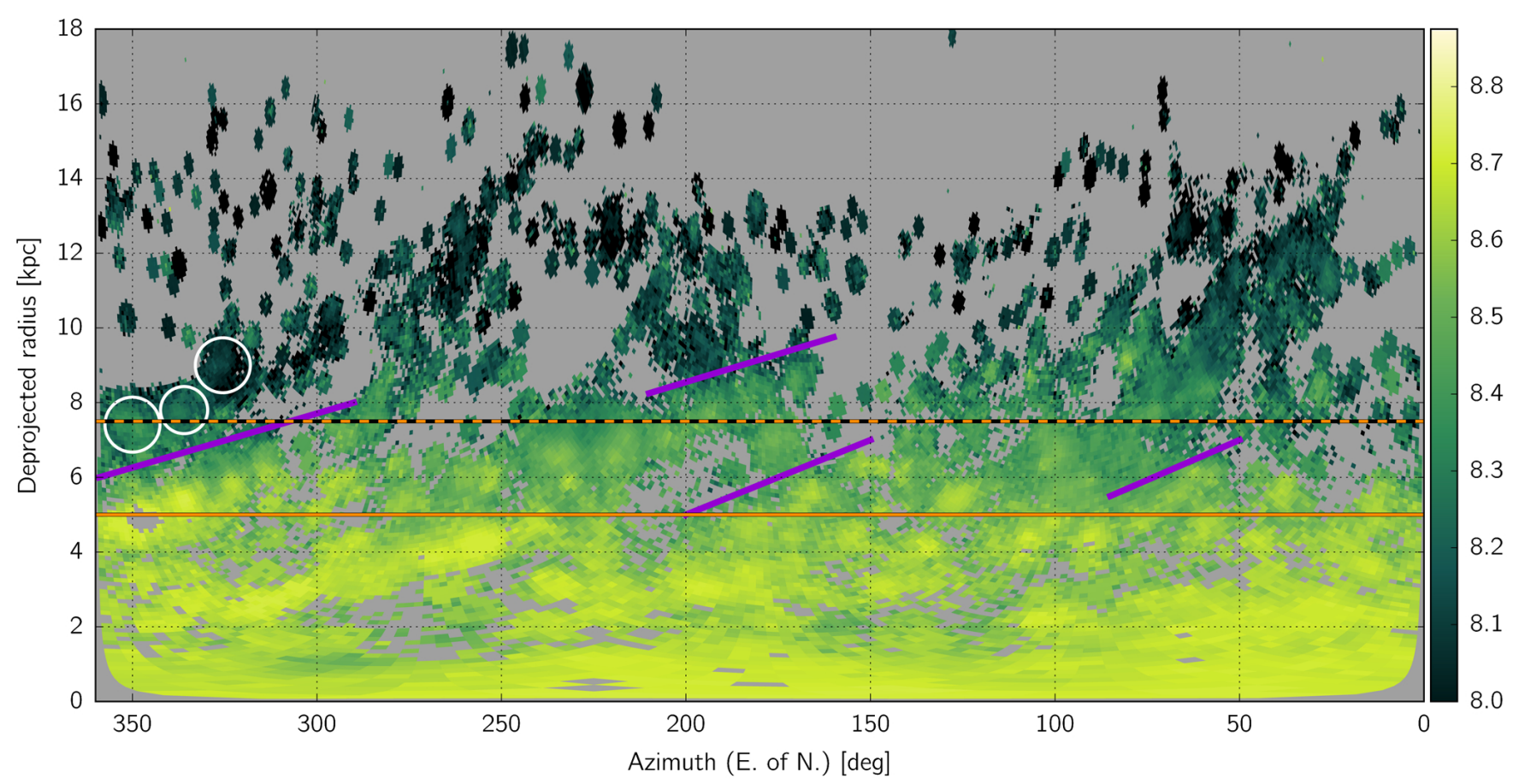

Fig. 10. De-wrapped abundance distribution of HCG 91c. Each spaxel in the datacube was reprojected in the azimuth-distance plane according to its location with respect to the galaxy center. For spaxels associated to a given aperture but without a reliable individual measure of $12+\log (\mathrm{O} / \mathrm{H})$, the aperture-derived value of $12+\log (\mathrm{O} / \mathrm{H})$ is shown instead. Spiral arms can be identified and tracked from 2 kpc outwards in this de-projected space. The iso-distance ellipses shown in the different panels of Figs. 7 and 8 become horizontal lines in this projection. Inclined purple lines mark the boundaries of spiral structures displaying a rapid variation of the oxygen abundance. The star-forming complexes found by Vogt et al. (2015) to have discrepant oxygen abundances with respect to their immediate surroundings are marked with white circles.

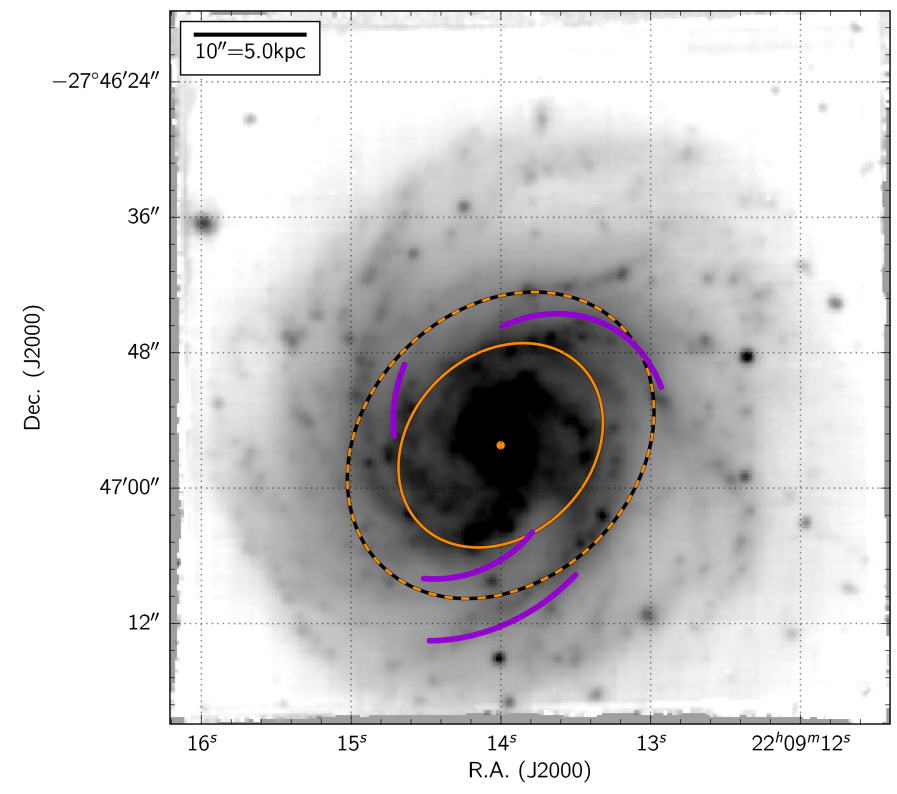

Fig. 11. White-light image of HCG 91c, reconstructed by collapsing the entire MUSE datacube. The galaxy center is marked with an orange dot. The projected effective radius $R_{\mathrm{e}}$ and $1.5 R_{\mathrm{e}}$ ellipses are shown in orange and dashed-orange, respectively. The location of the coherent, kpc-scale variations of the oxygen abundance traced in Fig. 10 are shown with purple curves. These effectively trace the edge of several of the spiral structures of HCG 91c.

instrument's unique combination of a large FoV and small spaxel size (soon to be fully exploited with the GALACSI ground-layer adaptive optics system and the Four-Laser Guide Star Facility, see Stuik et al. 2006; Bonaccini Calia et al. 2014) is effectively pushing the distance (out to $\sim 100 \mathrm{Mpc}$ ) at which abundance maps can both cover the optical discs of galaxies out to $\geq 2 R_{\mathrm{e}}$ and resolve sub-kpc structures. Such targets form the ideal link between projects targeting extremely nearby systems down to pc-scales (e.g., Kreckel et al. 2016) and those exploring the highredshift Universe: provided observations are performed under excellent seeing conditions, and with exposure times suitable to detect all strong emission lines with sufficient $\mathrm{S} / \mathrm{N}$ to characterize $\mathrm{H}$ II regions up to $2 R_{\mathrm{e}}$ and beyond.

Acknowledgements. We thank the anonymous referee for a prompt and constructive report. This research has made use of BRUTUS, a Python module to process datacubes from integral field spectrographs hosted at http://fpavogt. github.io/brutus/. BRUTUS relies on STATSMODEL (Seabold \& Perktold 2010), PPXF (Cappellari \& Emsellem 2004), FIT_KINEMATIC_PA as described in Appendix C of Krajnović et al. (2006), MATPLOTLIB (Hunter 2007), ASTROPY, a community-developed core Python package for Astronomy (Astropy Collaboration et al. 2013), PHOTUTILS, an affiliated package of AsTROPY for photometry, APLPY, an open-source plotting package for Python hosted at http: //aplpy.github.com, MONTAGE, funded by the National Science Foundation under Grant Number ACI-1440620 and previously funded by the National Aeronautics and Space Administration's Earth Science Technology Office, Computation Technologies Project, under Cooperative Agreement Number NCC5-626 between NASA and the California Institute of Technology, and MPFIT, a Python script that uses the Levenberg-Marquardt technique (Moré 1978) to solve least-squares problems, based on an original FORTRAN code part of the MINPACK-1 package. This research has also made use of the ALADIN interactive sky atlas (Bonnarel et al. 2000), of SAOIMAGE DS9 (Joye \& Mandel 2003) developed by Smithsonian Astrophysical Observatory, of NASA's Astrophysics Data System, and of the NASA/IPAC Extragalactic Database (NED; Helou et al. 1991) which is operated by the Jet Propulsion Laboratory, California Institute of Technology, under contract with the National Aeronautics and Space Administration. Based on observations made with ESO Telescopes at the La Silla Paranal Observatory under programme ID 60.A-9317[A]. This work was co-funded under the Marie Curie Actions of the European Commission (FP7-COFUND). E.P. acknowledges support from the Spanish 
MINECO through project AYA2014-57490-P. L.V.M. acknowledges support from AYA2015-65973-C3-1-R and AYA2014-52013-C2-1-R grants (MINECO Spain/FEDER, UE).

\section{References}

Astropy Collaboration, Robitaille, T. P., Tollerud, E. J., et al. 2013, A\&A, 558 A33

Baba, J., Morokuma-Matsui, K., \& Egusa, F. 2015, PASJ, 67, L4

Berg, D. A., Skillman, E. D., Croxall, K. V., et al. 2015, ApJ, 806, 16

Bitsakis, T., Charmandaris, V., Appleton, P. N., et al. 2014, A\&A, 565, A25

Blanc, G. A., Weinzirl, T., Song, M., et al. 2013, AJ, 145, 138

Bonaccini Calia, D., Hackenberg, W., Holzlöhner, R., Lewis, S., \& Pfrommer, T. 2014, Adv. Opt. Technol., 3, 345

Bonnarel, F., Fernique, P., Bienaymé, O., et al. 2000, A\&AS, 143, 33

Borthakur, S., Yun, M. S., \& Verdes-Montenegro, L. 2010, ApJ, 710, 385

Bresolin, F. 2011, ApJ, 730, 129

Bresolin, F., Ryan-Weber, E., Kennicutt, R. C., \& Goddard, Q. 2009, ApJ, 695, 580

Calzetti, D., Armus, L., Bohlin, R. C., et al. 2000, ApJ, 533, 682

Cappellari, M., \& Emsellem, E. 2004, PASP, 116, 138

Childress, M., Vogt, F., Nielsen, J., \& Sharp, R. 2014a, Astrophysics Source Code Library [record ascl: 1402.034]

Childress, M. J., Vogt, F. P. A., Nielsen, J., \& Sharp, R. G. 2014b, Ap\&SS, 349, 617

Cid Fernandes, R., Pérez, E., García Benito, R., et al. 2013, A\&A, 557, A86

Cleveland, W. S. 1979, J. Am. Statist. Assoc., 74, 829

Croxall, K., Pogge, R. W., Berg, D. A., Skillman, E. D., \& Moustakas, J. 2016, ApJ, 830, 4

de Vaucouleurs, G., de Vaucouleurs, A., Corwin, Jr., H. G., et al. 1991, Third Reference Catalogue of Bright Galaxies. Vol. I: Explanations and References. Vol. II: Data for Galaxies between $0 \mathrm{~h}$ and 12h. Vol. III: Data for Galaxies between $12 \mathrm{~h}$ and $24 \mathrm{~h}$

Dobbs, C., \& Baba, J. 2014, Publ. Astron. Soc. Aust., 31, e035

Dopita, M., Hart, J., McGregor, P., et al. 2007, Ap\&SS, 310, 255

Dopita, M., Rhee, J., Farage, C., et al. 2010, Ap\&SS, 327, 245

Dopita, M. A., Sutherland, R. S., Nicholls, D. C., Kewley, L. J., \& Vogt, F. P. A. 2013, ApJS, 208, 10

Falcón-Barroso, J., Sánchez-Blázquez, P., Vazdekis, A., et al. 2011, A\&A, 532, A95

Fischera, J., \& Dopita, M. 2005, ApJ, 619, 340

Fitzpatrick, E. L. 1999, PASP, 111, 63

Freudling, W., Romaniello, M., Bramich, D. M., et al. 2013, A\&A, 559, A96

Girardi, L., Bressan, A., Bertelli, G., \& Chiosi, C. 2000, A\&AS, 141, 371

Grand, R. J. J., Kawata, D., \& Cropper, M. 2012, MNRAS, 421, 1529

Grand, R. J. J., Springel, V., Kawata, D., et al. 2016, MNRAS, 460, L94

Helou, G., Madore, B. F., Schmitz, M., et al. 1991, Databases and On-line Data in Astronomy, 171, 89

Ho, I.-T., Medling, A. M., Groves, B., et al. 2016, Ap\&SS, 361, \#280

Hunter, J. D. 2007, Comput. Sci. Eng., 9, 90

Joye, W. A., \& Mandel, E. 2003, in Astronomical Data Analysis Software and Systems XII, ASP Conf. Ser., 295, 489
Kaplan, K. F., Jogee, S., Kewley, L., et al. 2016, MNRAS, 462, 1642

Kennicutt, Jr., R. C., \& Garnett, D. R. 1996, ApJ, 456, 504

Kewley, L. J., \& Ellison, S. L. 2008, ApJ, 681, 1183

Krajnović, D., Cappellari, M., de Zeeuw, P. T., \& Copin, Y. 2006, MNRAS, 366, 787

Kreckel, K., Blanc, G. A., Schinnerer, E., et al. 2016, ApJ, 827, 103

Kroupa, P. 2001, MNRAS, 322, 231

Li, Y., Bresolin, F., \& Kennicutt, Jr., R. C. 2013, ApJ, 766, 17

López-Sánchez, Á. R., Dopita, M. A., Kewley, L. J., et al. 2012, MNRAS, 426, 2630

Luridiana, V., Morisset, C., \& Shaw, R. A. 2013, Astrophysics Source Code Library [record ascl: 1304.021]

Luridiana, V., Morisset, C., \& Shaw, R. A. 2015, A\&A, 573, A42

Marino, R. A., Rosales-Ortega, F. F., Sánchez, S. F., et al. 2013, A\&A, 559, A114

Mathewson, D. S., Hart, J., Wehner, H. P., Hovey, G. R., \& van Harmelen, J. 2013, J. Astron. History and Heritage, 16, 2

Moré, J. J. 1978, in Numerical Analysis: Proc. Biennial Conf. Held at Dundee, June 28-July 1, 1977 (Berlin Heidelberg: Springer), 105

Nicholls, D. C., Dopita, M. A., \& Sutherland, R. S. 2012, ApJ, 752, 148

Nicholls, D. C., Dopita, M. A., Sutherland, R. S., Kewley, L. J., \& Palay, E. 2013, ApJS, 207, 21

Nicholls, D. C., Sutherland, R. S., Dopita, M. A., Kewley, L. J., \& Groves, B. A. 2016, MNRAS, submitted

Rosales-Ortega, F. F., Kennicutt, R. C., Sánchez, S. F., et al. 2010, MNRAS, 405, 735

Rosales-Ortega, F. F., Díaz, A. I., Kennicutt, R. C., \& Sánchez, S. F. 2011, MNRAS, 415, 2439

Rosolowsky, E., \& Simon, J. D. 2008, ApJ, 675, 1213

Sánchez, S. F., Kennicutt, R. C., Gil de Paz, A., et al. 2012a, A\&A, 538, A8

Sánchez, S. F., Rosales-Ortega, F. F., Marino, R. A., et al. 2012b, A\&A, 546, A2

Sánchez, S. F., Rosales-Ortega, F. F., Iglesias-Páramo, J., et al. 2014, A\&A, 563, A49

Sánchez, S. F., Galbany, L., Pérez, E., et al. 2015, A\&A, 573, A105

Sánchez, S. F., Pérez, E., Sánchez-Blázquez, P., et al. 2016a, Rev. Mex. Astron. Astrofis., 52, 171

Sánchez, S. F., Pérez, E., Sánchez-Blázquez, P., et al. 2016b, Rev. Mex. Astron. Astrofis., 52, 21

Sánchez-Blázquez, P., Peletier, R. F., Jiménez-Vicente, J., et al. 2006, MNRAS, 371,703

Sánchez-Menguiano, L., Sánchez, S. F., Kawata, D., et al. 2016a, ApJ, 830, L40

Sánchez-Menguiano, L., Sánchez, S. F., Pérez, I., et al. 2016b, A\&A, 587, A70

Sanders, N. E., Caldwell, N., McDowell, J., \& Harding, P. 2012, ApJ, 758, 133

Schlafly, E. F., \& Finkbeiner, D. P. 2011, ApJ, 737, 103

Schlegel, D. J., Finkbeiner, D. P., \& Davis, M. 1998, ApJ, 500, 525

Seabold, S., \& Perktold, J. 2010, in Proc. 9th Python in Science Conf., 57

Stuik, R., Bacon, R., Conzelmann, R., et al. 2006, New Astron. Rev., 49, 618

Vazdekis, A., Sánchez-Blázquez, P., Falcón-Barroso, J., et al. 2010, MNRAS, 404, 1639

Vogt, F. P. A. 2015, PhD Thesis, Australian National University

Vogt, F. P. A., Dopita, M. A., Borthakur, S., et al. 2015, MNRAS, 450, 2593

Vogt, F. P. A., Seitenzahl, I. R., Dopita, M. A., \& Ruiter, A. J. 2017, PASP, 129, 058012

Zinchenko, I. A., Pilyugin, L. S., Grebel, E. K., Sánchez, S. F., \& Vílchez, J. M. 2016, MNRAS, 462, 2715 


\section{Appendix A: A brief description of BRUTUS}

Here, we briefly describe the principal processing steps performed by BRUTUS in the case of HCG 91c. The user interested in using this code should however bear in mind that the code is still under active development, and, as such, is subject to evolution over time.

BRUTUS is designed to require as little interaction as possible, in order to speed the processing of large (MUSE) datacubes. All parameters with scientific implications are fed by the user into the different routines using a dedicated input PICKLED file, thereby making the post-processing of a datacube largely hands-free. BRUTUS is able to exploit multiple CPUs to speed-up the processing of a given datacube, and aims at creating high-quality diagrams that fully exploit the world coordinate system (WCS) of the data. Other specificities of BRUTUS include the facts that: 1) it is entirely written in PYTHON, with no outside software required (with the exception of MONTAGE); 2) it is able to cope with spectra with a non-uniform spectral resolution, as is the case for the MUSE instrument; 3) it allows both a spaxel-based processing or an aperturebased processing of a given datacube, with the in-built ability to identify structures in the data (e.g., H II regions) and create dedicated apertures, and 4) it exploits existing PYTHON tools with proven track-records developed by the community and individuals, including ASTROPY (Astropy Collaboration et al. 2013), STATSMODELS (Seabold \& Perktold 2010), APLPY, PPXF (Cappellari \& Emsellem 2004) and FIT_KINEMATICS_PA (Krajnović et al. 2006).

Once again, we stress that BRUTUS is a work in progress, and should not yet be considered complete or fully stable; existing modules are being updated and new ones added depending on the specific needs of our team, and so is the link to additional useful community packages (e.g., PYNEB is a foreseen addition; Luridiana et al. 2013, 2015). Contributions from the community that help make the code more polyvalent, more reliable and/or expand its capabilities are also welcome through the Github interface. Finally, we note that BRUTUS is built to accommodate (in principle) data from any integral field spectrograph (both in the visible or in the IR), but it has, so far, been developed and tested with MUSE datacubes only.

\section{A.1. Continuum and emission line fitting}

After performing an initial measure of the $\mathrm{S} / \mathrm{N}$ in the continuum and $\mathrm{H} \alpha$ emission line for all spaxels in the datacube, each spaxel is corrected for the Galactic extinction along the line of sight towards HCG 91c. The Galactic extinction is derived via the Nasa Extragalactic Database (NED) from the Schlafly \& Finkbeiner (2011) recalibration of the Schlegel et al. (1998) infrared-based dust map; for HCG 91c, $A_{B}=0.069$ and $A_{V}=0.052$. The map is based on dust emission from COBE/DIRBE and IRAS/ISSA. The recalibration assumes a Fitzpatrick (1999) reddening law with $R_{V}=3.1$ and a different source spectrum than Schlegel et al. (1998).

Each spectrum with $S / N<3$ in the continuum is then fitted using the non-parametric Locally-Weighted Scatterplot Smoothing (LOWESS) technique (Cleveland 1979), via the NONPARAMETRIC.SMOOTHERS_LOWESS.LOWESS() routine inside the STATSMODELS module. This technique is ideal for fitting the stellar continuum at low $\mathrm{S} / \mathrm{N}$ when fitting stellar population models isn't reliable. The technique is robust against the presence of emission lines and/or bad pixels in the spectra, in that it can efficiently ignore them, using a multiple-pass approach.
We note that this technique ought to also be of interest for fitting the nebular continuum (as an alternative to parametric functions) for galactic targets such as planetary nebulae or H II regions (see e.g., Vogt et al. 2017, for the case of a supernova remnant).

The stellar continuum for all spaxels with $S / N \geq 3$ is fitted using the PPXF code (Cappellari \& Emsellem 2004). For such low $\mathrm{S} / \mathrm{N}$, great caution must be used when interpreting the scientific implications of the fit. In this article, we solely focus on the analysis of the strong emission lines in the datacube. Fitting the stellar continuum on a spaxel-by-spaxel basis allows us to derive a reasonable estimate of the amount of stellar absorption below the $\mathrm{H} \alpha$ and $\mathrm{H} \beta$ emission lines in the data absorption features which become visible at $S / N \cong 3$. Here, we fed PPXF with stellar population synthesis model predictions from the MILES stellar libraries (Sánchez-Blázquez et al. 2006; Falcón-Barroso et al. 2011) at $F W H M=2.5 \AA$, based on the code presented in Vazdekis et al. (2010), using a Kroupa revised IMF with slope 1.3 (Kroupa 2001), Padova+00 isochrones (Girardi et al. 2000), with 6 metallicity steps ranging from -1.71 to +0.22 , and 13 ages ranging from 0.0631 to 15.8489 Gyr. We experimented with different model parameters, and note that our final choice does not influence the results described in the article at any significant level.

Emission lines are fitted in a subsequent BRUTUS processing step, after subtracting the stellar continuum fitted either using the LOWESS algorithm (for spaxels with $S / N<3$ ) or PPXF (for spaxels with $S / N \geq 3$ ). We rely on the MPFIT module that uses the Levenberg-Marquardt technique (Moré 1978) to solve least-squares problems, based on an original Fortran code; part of the MINPACK-1 package. Specifically, we use the version of MPFIT included by M. Cappellari inside the Python package files of PPXF, but with a dedicated BRUTUS wrapper. The following strong emission lines are fitted simultaneously, all with the same (tied) velocity:

$$
G 1: \mathrm{H} \beta,[\mathrm{O} \mathrm{III}] \lambda 4959,[\mathrm{O} \mathrm{III}] \lambda 5007
$$

$$
G 2:[\mathrm{N} \text { II }] \lambda 6548, \mathrm{H} \alpha,[\mathrm{N} \mathrm{II}] \lambda 6583 \text {, }
$$$$
\text { [S II] }] 6716,[\mathrm{~S} \text { II] }] 26731 .
$$

The velocity dispersion was tied for all $G 1$ and $G 2$ lines respectively, as a consequence of the varying spectral resolution of MUSE. Whereas, in principle, BRUTUS can allow for all lines to be fitted with individual velocity dispersions, the time-cost is (at the time of submission of this article) prohibitive in practice, and the fit less reliable for low $\mathrm{S} / \mathrm{N}$ spectra. A single component fit is appropriate for all spaxels, as anticipated from the observations of HCG 91c acquired with a spectral resolution of $R=7000$ using the WiFeS integral field spectrograph (Dopita et al. 2007, 2010) as discussed in Vogt et al. (2015).

\section{A.2. Semi-automated detection of $H \|$ regions}

BRUTUS relies on the FIND_PEAKS() function inside the PHOTUTILS module to identify local maxima throughout the $\mathrm{H} \alpha$ emission flux map. To each local maxima, BRUTUS assigns a fixedsize aperture, with a radius set to $0.6 \operatorname{arcsec}=3$ spaxels. For most H II regions in HCG 91c, a fixed-size aperture is a suitable choice as the star-forming complexes are clearly distinguishable, but not individually resolved. Nonetheless, BRUTUS also offers the ability to interactively add or remove apertures of varying radii via an interactive MATPLOTLIB window. The ability to rapidly add or remove additional apertures with a simple mouse 
click allows to rapidly and efficiently refine the automated selection - necessarily imperfect - by removing spurious detections, adding missing apertures, and adjusting the aperture sizes for extended star-forming complexes. At the time of submission, this interactive inspection of the aperture list is the only (optional) manual step within BRUTUS. We stress that the interactivity of this step is a strong factor allowing one to speed-up the procedure; for the case of HCG 91c, revising (visually) the list of $556 \mathrm{H}$ II regions can be done in a matter of minutes.
From the list of apertures (i.e., their center and radius), a new data cube is generated, where each spaxel within a given aperture is replaced with the sum of all the spaxels inside that aperture. All other spaxels (not associated to any aperture) are left empty (i.e., as PYTHON's NANs). This results in a (somewhat redundant) cube that can be fed back into BRUTUS, and processed exactly as the normal cube - but with a crucial improvement in the $\mathrm{S} / \mathrm{N}$ associated with the fainter $\mathrm{HII}$ regions in the outer regions of the galaxy. 